ISSN: $1130-3743$

DOI: http://dx.doi.org/10.14201/teoredu2015271137153

\title{
DISEÑO DE RECURSOS TECNOLÓGICOS PARA LA FORMACIÓN CÍVICA Y ÉTICA: LA EXPERIENCIA DE LA TELESECUNDARIA EN MÉXICO
}

\author{
Technological resources design for ethical \& civic \\ education: the Telesecondary experience in Mexico
}

\section{Conception des ressources technologiques pour la formation civique et éthique: l'expérience au Telesecundaria en Mexique}

Leticia Gabriela LANDEROS AGUIRRE

Universidad Nacional Autónoma de México, Petén 499-201. Col. Vértiz Narvarte. Delegación Benito Juárez. C.P. O360. México, Distrito Federal.

lglanderos@yahoo.com.mx

Fecha de recepción: enero de 2015

Fecha de aceptación: abril de 2015

Biblid [(1130-3743) 27, 1-2015, 137-153]

\section{RESUMEN}

En el artículo se comparte la experiencia relativa al diseño de materiales educativos que apoyan la asignatura Formación Cívica y Ética (FCYE) en México. Se retoma el caso específico de la Telesecundaria, modalidad sustentada en el uso de tecnologías que atiende a más de un millón de estudiantes entre 12 y 15 años de edad en zonas semiurbanas y rurales del país. Se presentan una mirada sobre el proceso seguido en el año 2006 y algunos de los aspectos que resultaron más relevantes al articular una propuesta curricular con carácter nacional y una propuesta didáctica basada en el uso de tecnologías. Se destacan tres elementos: la definición de un 
posicionamiento teórico y didáctico sobre la formación cívica y ética; la definición de aprendizajes fundamentales a promover mediante los materiales diseñados, y los desafíos que conlleva generar e implementar recursos tecnológicos en contextos de exclusión social y amplia brecha digital.

Palabras clave: formación cívica y ética; telesecundaria; materiales educativos; recursos tecnológicos.

\section{SUMMARY}

This article shares the experience related to the design of training materials for the subject Civic E Ethical Education (CEEE) in Mexico. It refers back to the case of Telesecondary, a learning approach based on the use of technologies covering more than one million students from 12 to 15 years old in semi-urban and rural zones. It presents a view on the process followed in 2006 and some aspect that resulted to be the most relevant when articulating a curricular proposal to be used nationwide, as well as a didactical proposal based in the use of technologies. Three elements are highlighted: the development of a theoretical and pedagogical positioning about the civics \& ethics education, the definition of core learnings to be promoted throughout the designed materials, and the challenges that involves the generation and implementation of technological resources in contexts of social exclusion and a wide digital gaps.

Key words: Civic \& Ethics education; Telesecondary; training materials o learning materials; technological resources.

\section{SOMMAIRE}

Dans l'article on expose l'expérience sur le conception de matériaux éducatifs qui soutiennent le cours de Formation Civique et Morale (FCYE) au Mexique. On examine le cas spécifique de la Telesecundaria, modalité basée sur l'utilisation de technologies, qui s'occupe à plus de un million d'étudiants entre 12 et 15 années d'âge dans des zones semi-urbaines et rurales du pays. Il est étudié le analyse rapprochée durant lannée 2006 et certains des aspects qui se sont avérés plus significatifs, en articulant une proposition curriculaire à titre national et une proposition didactique basée sur lutilisation de technologies. Ils sont soulignés trois éléments: la définition d'une approche théorique et didactique sur la formation civique et morale, la définition dapprentissages fondamentaux qui doivent être promu au moyen des matériaux conçus, et des défis qui apparaissent en utilisant des ressources technologiques dans des contextes dexclusion sociale et avec un importante fossé numérique.

Mots clés: l'éducation civique et éthique, telesecundaria, matériel pédagogique, les ressources technologiques, apprentissage situé. 


\section{INTRODUCCIÓN}

Las siguientes páginas tienen la intención de ilustrar la relación entre nuevas tecnologías y aprendizajes cívico-éticos mediante una experiencia específica. Se trata de la construcción de materiales audiovisuales para la asignatura Formación Cívica y Ética en el sistema de Telesecundaria en México. El trabajo ha sido elaborado desde la perspectiva de una integrante del equipo autoral y tiene el propósito de asentar parte del proceso seguido así como algunas reflexiones hechas a la distancia.

Su contenido está organizado en tres bloques. El primero de ellos, a modo de referente para el lector, incluye algunos datos sobre el sistema de Telesecundaria y sobre la asignatura Formación Cívica y Ėtica; ambos elementos constituyen parte de la superficie en la que se inscribe la experiencia. El segundo momento alude a los referentes usados para el diseño de los materiales y a la función pedagógica que se pensó para ellos. La intención es mostrar la importancia de una postura clarificada respecto a la formación cívica y ética, como eje que dote de sentido y vertebre cualquier diseño y uso de tecnologías. La última parte está dedicada a enunciar algunos de los desafíos que este proceso implicó. Dada la brevedad del espacio se apuntan sólo algunas ideas que den cuenta de los muchos aspectos implicados al momento de articular aprendizajes cívico-éticos y nuevas tecnologías. Para el caso de México y de la telesecundaria, esto incluyó enfrentarse a la implementación en contextos sociales y tecnológicos desfavorables y con escasas condiciones institucionales.

\section{REFERENTES PARA LA EXPERIENCIA}

\subsection{La formación cívica y ética. El escenario curricular}

La formación cívica ha estado presente de maneras diversas en las propuestas educativas y curriculares del México moderno; no obstante, el año 1999 constituye un momento particularmente relevante por la creación de la asignatura Formación Cívica y Ética en la educación secundaria1. Con este espacio se intentó superar los planteamientos de la asignatura existente hasta entonces denominada Educación Cívica, cuyo perfil corresponde con lo que algunos estudiosos del tema han denominado 'el civismo clásico' (Latapí, 2003; Conde y Canedo, 2004); esto es: un estudio centrado en el aprendizaje de la organización política de México, con una perspectiva básicamente jurídica sobre los derechos, con mínima alusión a procesos y principios democráticos, y con una orientación pedagógica centrada en el plano conceptual.

1. En México, la secundaria es el tercer nivel de educación básica, y va aproximadamente de los 12 a los 15 años de edad. Le anteceden los niveles de preescolar y primaria. Hasta el ciclo escolar 2011-2012, los registros indican más de 6 millones de alumnos cursando estudios secundarios. 
Si bien la propuesta de Formación Cívica y Ética amerita por sí misma un análisis crítico sobre su contenido, enfoque y evolución en los años recientes, vale decir que en su momento constituyó un salto cualitativo respecto al modo de concebir la formación ética y ciudadana en México. Por ejemplo, bajo esta nueva denominación se habló por primera vez de la necesidad de un enfoque 'formativo', que apelara no sólo a la construcción de saberes conceptuales sino que incentivara procesos de desarrollo sociomoral en niños y jóvenes; se introdujeron elementos de una democracia participativa y se apeló a métodos cercanos a la vivencia y el desarrollo del juicio moral.

La investigación educativa en la última década ha dado cuenta de que la llegada de esta asignatura no generó cambios sustanciales en las prácticas escolares; sin embargo, esta nueva forma de enunciación, y su legitimación a través de un currículum nacional, permitió la entrada de debates sobre el significado de formar cívica y éticamente, el papel de la escuela en ello y las herramientas pedagógicas requeridas.

Sobre lo primero (¿qué significa formar cívica y éticamente?) en el siguiente cuadro puede verse un ejemplo de las variaciones de sentido que se han expresado en los programas de estudio, y sus distintos puntos de anudaje en los últimos quince años. Como puede observarse, estos transitan desde una visión centrada en la información y una idea de ciudadanía sólo como condición futura, hacia otra orientada al desarrollo de competencias cívicas y éticas y la atención a desafíos sociales presentes. 


\section{ImAgen 1. Propósitos Del CivisMo y la Formación CíviCA y ÉTICA (SEP, 1993, 2000, 2006, 2011)}

\begin{tabular}{|c|c|}
\hline $\begin{array}{c}\text { VERSIÓN DEL } \\
\text { PROGRAMA DE ESTUdios }\end{array}$ & PropósITOS \\
\hline Civismo 1993 & $\begin{array}{l}\text { Ofrecer a los alumnos... las bases de información y orientación } \\
\text { sobre sus derechos y sus responsabilidades, relacionados con } \\
\text { su condición actual de adolescentes y también con su futura } \\
\text { actuación ciudadana. }\end{array}$ \\
\hline $\begin{array}{l}\text { Formación Cívica y } \\
\text { Ética } 1999\end{array}$ & $\begin{array}{l}\text {... Proporcionar elementos conceptuales y de juicio para que los } \\
\text { jóvenes desarrollen la capacidad de análisis y discusión necesaria } \\
\text { para tomar decisiones personales y colectivas que contribuyan al } \\
\text { mejoramiento de su desempeño en la sociedad. }\end{array}$ \\
\hline $\begin{array}{l}\text { Reforma curricular } \\
2006\end{array}$ & $\begin{array}{l}\text {... Apunta al desarrollo de competencias, es decir, conjuntos de } \\
\text { nociones, estrategias intelectuales, disposiciones y actitudes que } \\
\text { permitan a los estudiantes responder ante situaciones de su vida } \\
\text { personal y social, en las que se involucra su perspectiva moral y } \\
\text { cívica: al actuar, tomar decisiones, elegir entre opciones de valor, } \\
\text { encarar conflictos y participar en asuntos colectivos. }\end{array}$ \\
\hline $\begin{array}{l}\text { Reforma curricular } \\
2011\end{array}$ & $\begin{array}{l}\text { La asignatura de Formación Cívica y Ética se concibe como } \\
\text { un conjunto de experiencias organizadas y sistemáticas que } \\
\text { contribuyen a formarcriterios y a asumirposturas y compromisos } \\
\text { relacionados con el desarrollo personal y social de los alumnos, } \\
\text { teniendo como base los derechos humanos y los principios } \\
\text { democráticos. }\end{array}$ \\
\hline
\end{tabular}

Evidentemente, cada una de estas formas de definir la formación cívica y ética es la expresión de posturas y debates ligados al momento histórico (institucional, académico, incluso político del país) en que se gestaron. En la primera versión de 1999 el enfoque se orienta principalmente hacia la formación en valores y la selección de contenidos recupera mucho de la perspectiva del desarrollo humano: intereses de los jóvenes, desafíos de la adolescencia, toma de decisiones profesionales y proyecto de vida. En sus versiones posteriores, ganan terreno los contenidos propiamente cívicos y un enfoque de 'desempeño' social bajo principios democráticos. En este marco, a partir de la reforma curricular de 2006, el programa de estudios se configura en torno a ocho competencias eje de la formación cívica y ética²:

2. En la posterior reforma curricular de 2011 se mantuvo el enfoque por competencias con algunas variaciones en los contenidos específicos. A partir de 2014 la Secretaría de Educación Pública ha iniciado un nuevo proceso de ajuste que impactará en la perspectiva y contenidos de esta asignatura. La organización basada en ocho competencias es la vigente en el ciclo escolar 2014-2015. 
- Conocimiento y cuidado de sí mismo

- Autorregulación y ejercicio responsable de la libertad

- Respeto y valoración de la diversidad

- Sentido de pertenencia a la comunidad, la nación y la humanidad

- Manejo y resolución de conflictos

- Participación social y política

- Apego a la legalidad y sentido de justicia

- Comprensión y aprecio por la democracia

Justo en ese marco (2006) es que se instala la experiencia específica que se desea compartir aquí. Debe verse por tanto como una práctica situada en un cierto escenario curricular de reforma, pero también en el marco de un proceso de ajustes constantes en los significados y énfasis sobre la vida en el espacio público. En ese año, la formación cívica y ética da un giro respecto a su versión inicial al alinearse al enfoque por competencias. En el programa se conservan algunos elementos originales, pero a la vez se instalan otros nuevos como la propia noción de competencias, un mayor énfasis en la democracia y en ciertos procedimientos como el diálogo.

\subsection{La Telesecundaria y el uso de tecnologías}

Un segundo componente para situar la experiencia tiene que ver con su ámbito de aplicación; en este caso, las escuelas telesecundarias en México.

La Telesecundaria es una modalidad educativa creada en 1968 como una alternativa al rezago educativo, a la dificultad geográfica que impedía llegar a la población en zonas lejanas en un país tan amplio y complejo como México y a la carencia de recursos para atender las necesidades educativas de todos (SEP, 2010, 7). Constituye una de las cinco modalidades de la educación secundaria, junto con las escuelas generales, técnicas, comunitarias y para trabajadores. Actualmente, la Telesecundaria atiende a más de un millón de estudiantes en todo el país, principalmente en zonas rurales y semiurbanas.

Aunque no es exclusiva de sectores marginados, sí puede decirse que una amplia proporción de los niños que asisten a las escuelas de esta modalidad provienen de sectores en desventaja social y viven en condiciones de pobreza. 


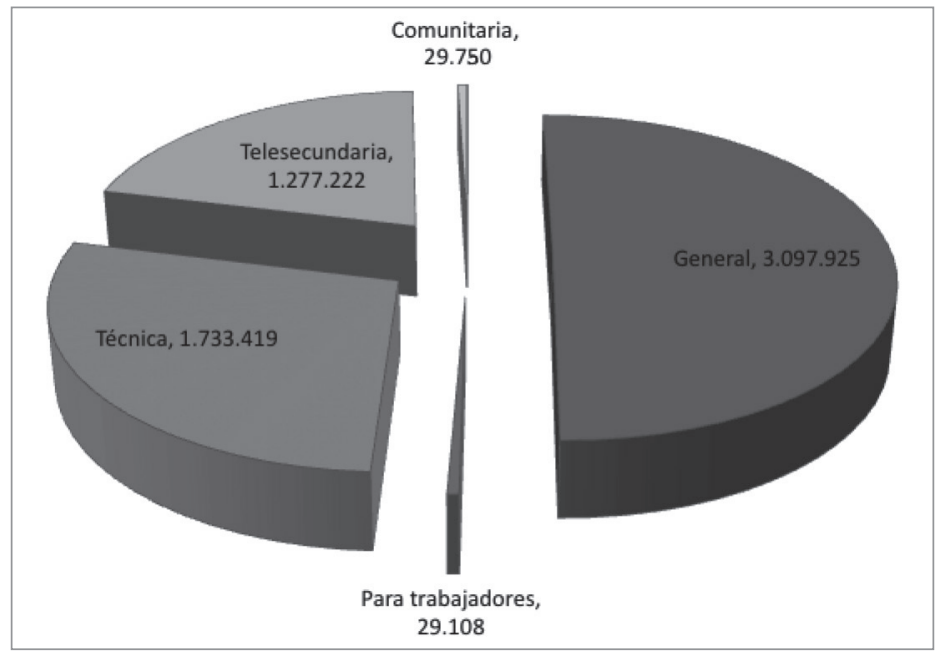

En parte por ello su enfoque inicial retomó algo de las experiencias de la educación rural en México, para sumarlo a los resultados del desarrollo científico y tecnológico de la época. En aquel momento, su propuesta pedagógica se basaba en la existencia de dos figuras de apoyo (el telemaestro y maestro-monitor) y dos recursos (televisión y guías de estudio). La dinámica generada con ello no resultaba muy distinta a una clase tradicional: un profesor que impartía clases a través de los programas de televisión y otro que apoyaba a los estudiantes en la resolución de ejercicios contenidos en las guías de estudio. A diferencia de las secundarias regulares que cuentan con un profesor por cada asignatura, las telesecundarias tenían (y tienen hasta la fecha) uno solo, que apoya a todo el grupo en todas las asignaturas.

Este modelo ha tenido cambios tanto en su enfoque como en el conjunto de recursos de los que se apoya. El más reciente de ellos sucede también en torno a la Reforma Integral de la Educación Secundaria (2001-2006) e implicó la construcción de un nuevo modelo para la renovación de la telesecundaria en torno a cinco principios (SEP, 2011, 13):

- El alumno como gestor de su propio aprendizaje.

- Las Tecnologías de la Información y la Comunicación (TIC) como apoyo indispensable para el aprendizaje del alumno.

- El aprendizaje a partir de situaciones reales.

- El desarrollo de proyectos productivos comunitarios.

- El trabajo colaborativo. 
Este nuevo modelo incluyó el replanteamiento del sentido y características de los materiales educativos, bajo un nuevo esquema que integraba ya no sólo la televisión sino un conjunto de recursos, entre ellos:

- Libros de texto para alumnos y profesores.

- Materiales interactivos que podían ejecutarse en las computadoras disponibles para los alumnos a través de un aula de medios, o bien, mediante un pizarrón electrónico.

- Programas de televisión transmitidos vía satélite a las escuelas a través del Sistema EDUSAT, perteneciente a la Secretaría de Educación Pública.

- Videos a disposición de los docentes para ser usados en el momento que los requieran.

En ese marco es que la Secretaría de Educación Pública de México, por convenio con el Instituto Latinoamericano para Comunicación Educativa (ILCE), convocó a equipos de especialistas para el diseño de los nuevos materiales educativos de Telesecundaria. Para el caso de Formación Cívica y Ética por vez primera se contó con estos recursos, integrados por un libro para el alumno, un libro para el maestro y más de cincuenta materiales audiovisuales para el tratamiento de los contenidos de los programas de estudios ${ }^{3}$.

\section{3. ¿TECNOlogías Para QUÉ?: El DESARROllo DE CAPACIDADES CÍVICO-ÉTICAS}

Para quienes conformamos los equipos autorales ${ }^{4}$ su elaboración constituyó un proceso complejo en varios sentidos; dos de los principales: construir una propuesta pedagógica con carácter nacional para el aprendizaje cívico ético (cosa en sí misma desafiante si se piensa en un país con la extensión, diversidad y complejidad de México) y hacer que esa propuesta integrara congruentemente el uso de nuevas tecnologías.

Algunos aspectos de la tarea se aprendieron sobre la marcha, y se resolvieron con distintos niveles de calidad, pero una mirada a la distancia permite reconocer algunos elementos que resultaron centrales y de los cuales recupero aquí sólo dos:

\subsection{Clarificar la postura sobre el sentido de la FCYE}

Un aspecto relevante para la construcción de la propuesta y los materiales fue la definición de la postura desde la cual serían pensados. Para entonces,

3. El programa de asignatura y los contenidos específicos pueden ser consultados en: http:// basica.sep.gob.mx/reformaintegral/sitio/pdf/secundaria/plan/FCyESec11.pdf.

4. Para el caso de Formación Cívica y Ética, éste estuvo integrado por Liliana Poveda, Concepción Chávez y Leticia Landeros, así como un equipo responsable del desarrollo tecnológico de los recursos, coordinado por Karla Pedroza. 
diversos estudios (Schmelkes, 2004; Latapí, 2003) habían dado cuenta de que bajo denominaciones similares (valores, civismo, paz, legalidad) circulaban en México programas educativos con posturas contrastantes e incluso antagónicas, algunas de las cuales desconocían la libertad de los sujetos y se orientaban más bien hacia el adoctrinamiento.

La existencia del programa de estudios para formación cívica y ética, con un enfoque democrático, nacional y formativo, brindó algunos criterios para mirar críticamente estos programas y delimitar lo que resultaba congruente o no con los propósitos establecidos. Sin embargo, las orientaciones didácticas marcadas por los programas eran -y son- de orden más bien general; por tanto, las posibilidades para concretar los contenidos seguían siendo diversas y con cierta cabida para la contradicción. La investigación educativa de esos años y posterior ha mostrado cómo esto se amplía al llegar al aula, donde los docentes de la asignatura toman sus propias posturas ante los contenidos cívico-éticos, eligen métodos y establecen prioridades (Yurén, 2007; COMIE, 2013; Rodríguez, 2009).

En este contexto, había que entender que los materiales educativos para Telesecundaria representaban una mediación entre el plano general de los programas y las decisiones didácticas específicas de cada docente. De ahí la importancia de enfrentar el diseño haciendo explícitas las posturas asumidas por el equipo autoral y por tanto el lugar desde donde se hacía la traducción de los programas de estudio a los materiales.

Las miradas nunca fueron plenamente uniformes, lo cual dio una especial riqueza al proceso, pero a la vez fue posible identificar principios e ideas eje sobre las que había acuerdo; por ejemplo: los aprendizajes cívicos y éticos tenían que verse desde un punto de vista formativo; es decir, que se apelara a procesos en los que los sujetos se configuraran a sí mismos y se asumieran como creadores o renovadores de la cultura y los órdenes sociales (Yurén, 2000, 29). Esto obligaba a incluir un componente constructivo y transformador. De igual forma se asumía la postura de que el desarrollo sociomoral constituye parte fundamental de este proceso formativo (Hersch, Reimer y Paolitto, 1998), que éste debe promover la autonomía de los sujetos y su capacidad para enfrentar desafíos y tensiones de la vida en sociedad.

A este posicionamiento pedagógico sobre el sentido de la formación se integró un elemento más: la incorporación de los derechos humanos y la democracia como nociones articuladoras de sentido. De hecho, en buena medida la formación cívica y ética era entendida por el equipo autoral como una educación en derechos humanos y como una vía para aprender en la práctica los principios de una democracia participativa. En congruencia con ello, la propuesta en su conjunto debía abordar elementos tales como: la construcción de sujetos de derechos, la promoción de la equidad, la puesta en evidencia de condiciones que vulneran derechos, la generación de oportunidades para el ejercicio de la participación, la práctica de la libre expresión mediante el debate y la argumentación. Si bien la idea de educar en valores seguía presente, la propuesta apostaba más por una formación sociomoral y con los derechos humanos y la democracia como referentes éticos y políticos. 
Dicho enfoque implicó al mismo tiempo optar por ciertos métodos para el trabajo en el aula, que fueron retomados principalmente de las propuestas educativas desarrolladas por organizaciones no gubernamentales y grupos académicos en Iberoamérica desde los años 90. Esto incluía reconocer la herencia generada por grupos de educación popular, educación en derechos humanos y educación para la paz tanto en México como en Uruguay, Chile, Colombia, España, y otros más. De Latinoamérica se retomó de forma especial la perspectiva problematizadora y de derechos humanos, gestada en países que, como Chile, vivieron en contexto de dictadura militar y represión (Magendzo, 2008). Ésta obligaba a una mirada crítica de la realidad y a llevar al aula las tensiones existentes en contextos de violencia (estructural o directa), de pobreza o de condiciones sociales y políticas de exclusión, comunes en nuestros países. De España, se integraron un conjunto de planteamientos teóricos y métodos pedagógicos para la educación moral y en valores, que apostaban por el desarrollo de la autonomía. El enfoque socioafectivo y técnicas como la clarificación de valores y la discusión de dilemas (Buxarrais, Martínez, Puig y Trilla, 1997) fueron centrales, en tanto ayudaron a poner en duda la perspectiva fundamentalmente conceptual que predominó en México hasta 1999, y a hacer evidentes los procesos personales y colectivos sobre los que la escuela debía incidir ${ }^{5}$.

De entrada, esta selección ya imponía un cierto perfil a la tarea: aquello que se construyera debía incentivar la idea ya citada sobre la formación, motivar el ejercicio de la autonomía moral, la participación y el compromiso con la transformación social en favor de los derechos humanos.

\subsection{Reconocer distintos aprendizajes susceptibles de promoverse a través de tecnologías}

Un plano más del proceso fue el relativo a la función de los recursos y materiales, particularmente aquellos referidos a las nuevas tecnologías (interactivos, videos): ¿cuál sería su lugar en el conjunto de la propuesta didáctica?

Las respuestas generadas tuvieron varias mediaciones. Una de ellas, desde luego, fue el posicionamiento autoral explicado anteriormente, que por sí mismo

5. Para la propia Secretaría de Educación Pública (no sólo para los diseñadores de libros de texto), la experiencia española fue uno de los principales referentes teóricos y metodológicos al configurar el primer programa de Formación Cívica y Ética. Obras como la citada aquí de BuXARRAIS, MARTínez, Puig y Trilla, conocida en México como "La educación moral en primaria y en secundaria. Una experiencia española", y "El contrato moral del profesorado" (MARTínEZ, 1998) fueron dos de los pocos materiales que durante años existieron sobre el tema en la Biblioteca para la Actualización del Maestro de la SEP. Actualmente este acervo se ha ampliado, pero al menos durante la primera década de existencia de la asignatura los planteamientos asentados en estos textos tuvieron un lugar hegemónico en el discurso de autoridades y docentes, marcando prácticas. A la fecha, varios de esos elementos (fundamentalmente los referidos a los métodos de trabajo en el aula) se han sedimentado y se mantienen como parte del enfoque se enseñanza en los programas de estudio. 
ya marcaba una intención didáctica a los recursos. Pero había más. Otra de ellas fue el programa de estudios establecido por la Secretaría de Educación, el enfoque ahí plasmado y la selección de contenidos que normativamente debían ser desarrollados. No en vano se trataba de una tarea institucional, con carácter nacional, y que debía atender una propuesta curricular que es igual para todos los estudiantes del país. En este caso, vale decir que la articulación entre posicionamiento autoral y currículum no estuvo libre de tensiones, pero resultó posible. A diferencia de versiones posteriores del programa de estudios, el de 2006 tenía un planteamiento más ordenado, menos saturado y con mayor congruencia interna (pese a sus redundancias e inconsistencias), lo cual permitía dar seguimiento a ciertos procesos clave para la formación cívica y ética. Entre ellos estaban entre otros el diálogo, el juicio moral autónomo y el análisis crítico de la realidad, además de la construcción de nociones clave sobre la democracia y los derechos humanos.

Todos los materiales se construyeron teniendo como pauta los programas y contenidos, pero algunos de ellos, particularmente los interactivos, sirvieron como herramienta para impulsar estos procesos clave de manera transversal y rebasando el tratamiento literal del programa. Con fines de ilustración se muestran aquí algunos ejemplos de estos recursos y de la forma en que recurrió a ellos didácticamente ${ }^{6}$ :

\subsubsection{El desarrollo del juicio moral}

Con base en las propuestas de L. Kolhberg sobre este tema, la incorporación de nociones como el 'juicio prudencial' (Yurén, 2005, 27-28) y el aprendizaje a través de problemas (Magendzo, 2005) se desarrollaron materiales que invitaran a los estudiantes a plantearse situaciones dilemáticas reales o ficticias.

Los materiales interactivos (elaborados a modo de juegos) resultaron especialmente valiosos para este fin. En ellos se planteaban situaciones cuya resolución obligaba una reflexión ética personal y una toma de postura: había que decidir qué hacer. Esta elección tenía que ser mediada también por el diálogo con el grupo, de modo que fuera posible conocer otros puntos de vista, argumentar y revisar la propia postura.

6. Las referencias e imágenes incluidas a continuación fueron tomadas de los materiales originales propiedad de la Secretaría de Educación Pública y el Instituto Latinoamericano de la Comunicación Educativa. 
IMAGEN 3. INTERACTIVO ¿Y TÚ QUÉ HARÍAS? (SEP, 2006B, MATERIAL EN LÍNEA)
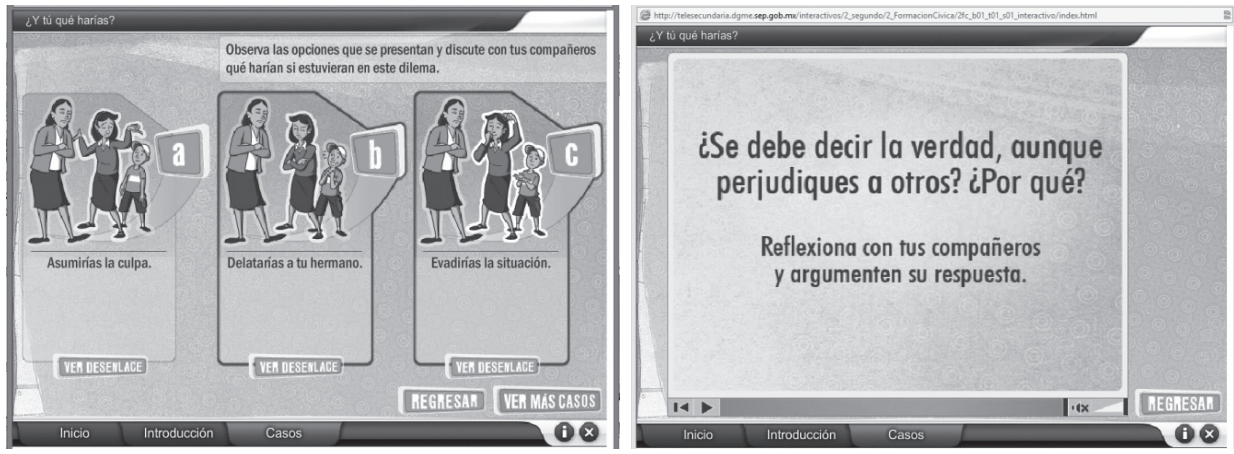

Los ejercicios no fueron diseñados con respuestas únicas o 'correctas', sino que dejan ver que cada elección tiene consecuencias. Incluso aquella que consideramos éticamente correcta puede traer consigo implicaciones que hay que asumir y enfrentar. Un ejemplo: ser testigo de una falta cometida por alguien a quien quieres y deseas proteger. ¿Es correcto delatar? Más allá de inducir una respuesta obvia o 'correcta', la finalidad del material es motivar un proceso de reflexión y reconocer matices en la realidad. Una vez que el estudiante (individualmente o con su grupo) elige la reacción más cercana a lo que haría en un caso así, el interactivo le despliega una posible consecuencia que no siempre es del todo agradable y que le pone nuevamente en tensión: ¿mantendría mi decisión?, ¿la cambiaría según la consecuencia?

Ejercicios como éste recuperan la estructura ya sabida sobre la discusión de dilemas morales (Buxarrais, Martínez, Puig y Trilla, 1997, 99) para aplicarla a un recurso tecnológico y servir como detonante para la reflexión moral y para el diálogo grupal.

\subsubsection{Pensamiento crítico}

Éste resulta un aprendizaje fundamental desde una perspectiva de derechos humanos en tanto abre posibilidades para ver más allá de lo evidente, para establecer relaciones complejas y para reconocer situaciones que vulneran los derechos de personas y grupos (Cullen, 2004).

En este punto, los videos y los interactivos fueron la vía, por ejemplo, para debatir un tema controvertido y escuchar distintos puntos de vista, para dramatizar una situación que luego pudiera ser discutida o para hacer que los estudiantes tomaran parte de problemas de la localidad y reconocieran los factores que los influyen. Algunas de estas situaciones podían ser hipotéticas, pero daban pie para retomarlas en el salón de clases e introducir otras sucedidas en la comunidad donde se ubica cada escuela. Por ejemplo, en la actividad que se ilustra más abajo 
denominada "Descubro al responsable", el propósito central es analizar un problema comunitario y ser capaces de reconocer la responsabilidad de cada miembro de la sociedad, tanto con el problema como en la generación de soluciones.

IMAGEN 4. INTERACTIVO DESCUBRO AL RESPONSABLE (SEP, 2006B, MATERIAL EN LÍNEA)
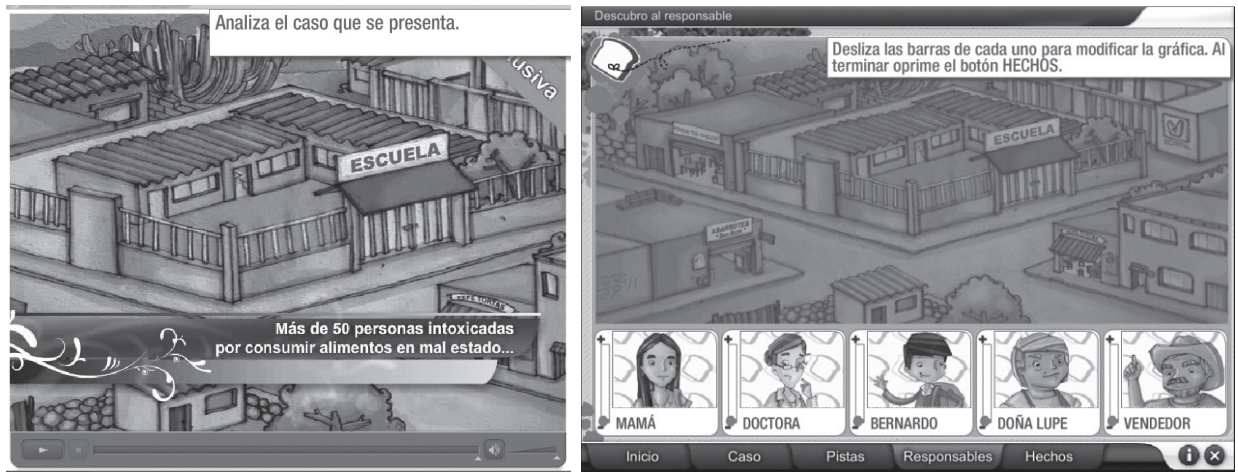

Entre otras cosas, esto permite mostrar que un hecho social no es la consecuencia lineal de un solo factor, sino que se configura a partir de actos y condiciones diversas en las que participamos los sujetos.

\subsubsection{El aprendizaje de nociones y procedimientos fundamentales}

Un enfoque formativo incluye también el trabajo en torno a nociones y procedimientos que requieren ser aprendidos, por ejemplo, para la construcción de una sociedad democrática. Por ello, una parte de los materiales están también dedicados a ello, procurando mantener un vínculo con la acción. Es decir, las nociones no sólo son parte de un bagaje intelectual sino que resultan necesarias para enfrentar situaciones y resolver problemas. En ese sentido, su aprendizaje requiere situarse en el marco de una experiencia.

Para el caso de secundaria (y telesecundaria) un elemento de esta experiencia es la elección de Consejos de alumnos. Se trata de una figura de representación estudiantil legitimada en las escuelas, aunque en la práctica no cuenta con poder de interlocución ni toma de decisiones y se les ha limitado a tareas irrelevantes como la organización de festejos o ceremonias predefinidas por el cuerpo docente. Pese a ello, la existencia de esta figura y la realización de procesos de elección constituyen una valiosa oportunidad para el aprendizaje de nociones y procedimientos para la democracia. 
IMAGEN 5. INTERACTIVO VAMOS A VOTAR (SEP; 2006B, MATERIAL EN LÍNEA)
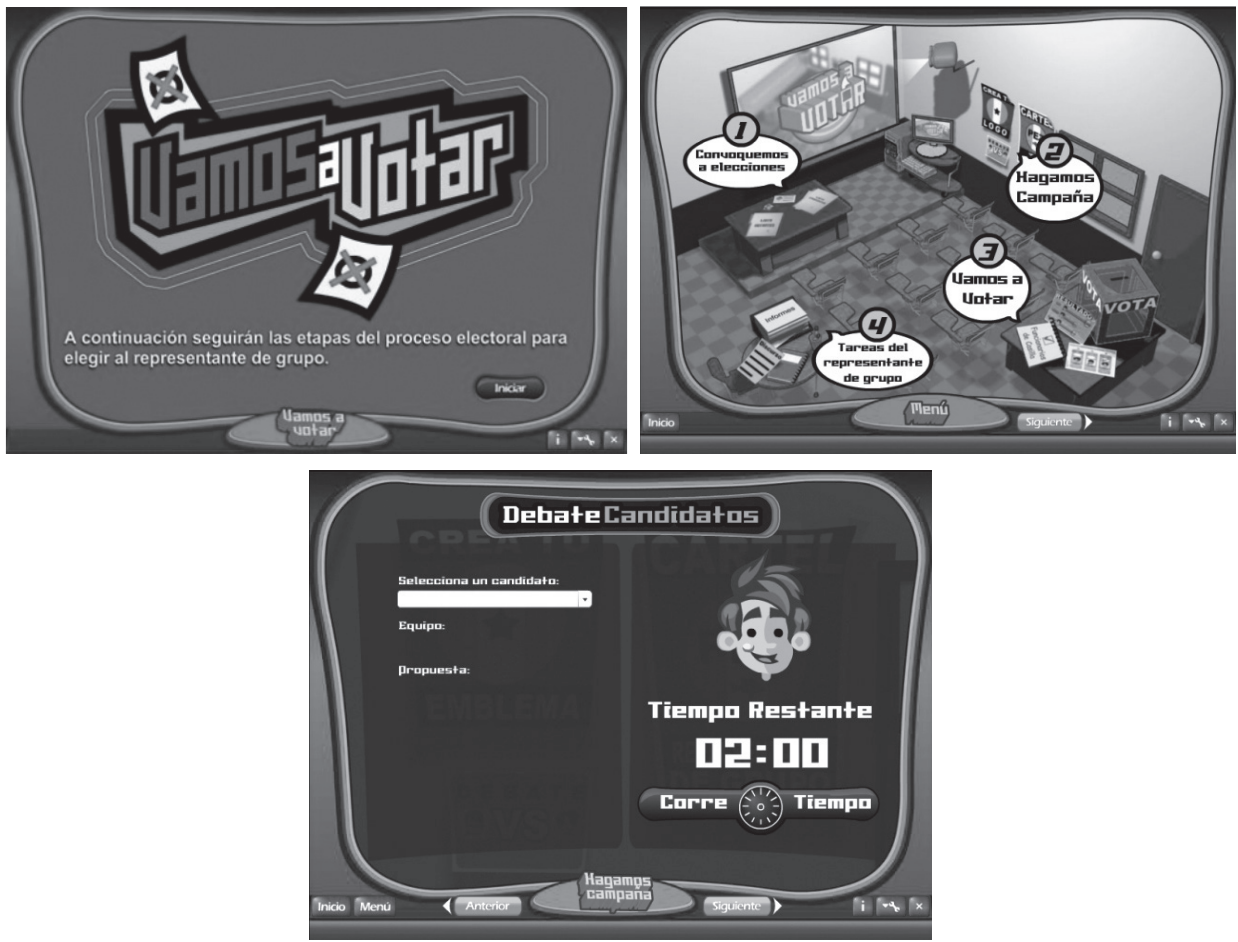

Así, una parte de los materiales audiovisuales diseñados da elementos para ubicar los pasos de un proceso electoral, organizarse para integrar planillas, elaborar propuestas, debatirlas y argumentar, y dar seguimiento a las "promesas de campaña'. En el ejemplo que se muestra ("Vamos a votar"), el recurso tiene más de una función: contiene información sintética que recuerda a los alumnos cada paso en un proceso de elección y su importancia para la democracia; pero, al mismo tiempo, ayuda a ponerlos en práctica durante la elección del Consejo de alumnos: permite asentar propuestas de las planillas, imprimir un ejemplo de urna para depositar los votos, regular el uso de la palabra en el debate de los candidatos mediante un cronómetro. Se trata de un material creado exprofeso para acompañar en aprendizajes fundamentales procedimentales que se articulan con el trabajo en los libros de texto y los videos para integrar también reflexiones éticas y valoraciones críticas sobre la democracia. 


\section{AlgunOS DESAFíos De LA EXPERIENCIA}

Lo expuesto hasta aquí refleja una parte del proceso: el relativo a la forma en que fueron pensados y fundamentados los materiales educativos para el aprendizaje cívico-ético en Telesecundaria. Sin embargo, el cierre de este trabajo está dedicado a citar algunos de los desafíos que este trabajo implicó. Cada uno de ellos invita a un desarrollo mucho más extenso, pero valga esta mención superficial para dar cuenta de la complejidad que conlleva el desarrollo de una propuesta educativa de este tipo y para este contexto específico de aplicación.

Entre estos desafíos se encuentran los siguientes:

- Construir una propuesta para contextos tan diversos, mayoritariamente en condiciones de exclusión social y a partir de un currículum nacional. Esto representa una tarea compleja cuyos retos no siempre es posible salvar. Vías para hacerlo fueron el hecho de diseñar creando oportunidades para que maestros y alumnos articularan con la realidad cercana y adaptaran las actividades a su contexto; y procurar la presencia de contextos diversos mediante la selección de casos y la propuesta visual. Aun así, es necesario reconocer la existencia de límites para acercarse al contexto de más de un millón de estudiantes en 32 entidades de la República Mexicana.

- Procurar congruencia entre la perspectiva formativa y el sentido de los recursos. Si bien a nivel autoral había claridad en la postura, el uso de nuevas tecnologías implicó establecer una relación con otras formas de lenguaje, con condiciones técnicas específicas, con modos de creación distintos. De esta relación dependía también la congruencia pedagógica de la propuesta, de modo que los materiales no se volvieran un fin en sí mismos ni un mero instrumento, sino parte integral de ella. En términos de habilidades, pensar en el diseño de material audiovisual era una novedad para parte del grupo, y requirió echar mano de (o construir) nuevas habilidades para lograr consistencia entre éstos y la postura asumida.

- Contar con recursos (bumanos, económicos, técnicos). Crear una propuesta basada en la combinación de tecnologías requiere echar a andar múltiples recursos. Algunos son de orden económico y son necesarios para desplegar todas las posibilidades que las nuevas tecnologías ofrecen. Otros son de tipo humano: contar con un equipo sensible al tema, dispuesto a aprender y cercano a una perspectiva compleja sobre la realidad social. En el caso de Telesecundaria la existencia de este equipo constituyó una ventaja, favoreció el diálogo entre lenguajes distintos y detonó el surgimiento de un grupo de profesionales de la comunicación interesados en la formación cívica y ética. A pesar de ello, la experiencia hizo evidente la escasa producción en la materia y la necesidad de desarrollar propuestas tecnológicas, visuales y artísticas para el aprendizaje en esta materia.

- Diseñar con nuevas tecnologías para contextos de exclusión y carencia. Si bien no correspondió al equipo de diseño la implementación, resultaba 
indispensable pensar en las condiciones técnicas de las escuelas en las que se utilizarían los materiales. Muchas de ellas sin luz, sin conexión a Internet o con conexión limitada, con escasa formación docente para el uso de tecnologías. Se crea una disyuntiva entre hacer de los materiales audiovisuales parte integral de la propuesta, y a la vez pensar que éstos tal vez no puedan ser utilizados por limitaciones técnicas. El desafío permanece y tiene que ver en buena medida con las condiciones de equidad y calidad del sistema educativo y del país en su conjunto. La propuesta de Telesecundaria, con todas sus virtudes, requiere de esfuerzos en todos los ámbitos para que los niños y niñas puedan disfrutar de ella.

El proceso hasta aquí descrito apunta algunos elementos que sobre los que valdría la pena profundizar, por varias razones. Una -de índole muy concreto e inmediato- es la reforma curricular en puerta y en cuyo marco ameritaría una evaluación de lo hecho hasta ahora. Otra más es el hecho de que lo expuesto en este escrito es apenas una parte de un proceso mucho más complejo que incluye a otros actores y espacios institucionales. No se conoce, por ejemplo, qué ha pasado con la implementación, cuál es el impacto de estos materiales y, en esa medida, qué tendría que conservarse o cambiarse.

Pero también vale la pena profundizar porque se trata de un terreno poco explorado. Hay mucho que indagar sobre el papel de las tecnologías para la formación cívica y ética, sobre los apoyos que pueden brindar para el desarrollo de los diversos aprendizajes que ésta incluye y sobre las necesidades de formación docente que detonan. Para el caso de la Telesecundaria, avanzar en ello puede ser una vía para fortalecer la calidad de la educación que reciben más de un millón de adolescentes en México, en la única modalidad educativa vinculada desde su origen al uso integral de nuevas tecnologías.

\section{REFERENCIAS BIBLIOGRÁFICAS}

Buxarrais, M. R.; Martínez, M.; Puig, J. M. y Trilla, J. (1997) La educación moral en primaria y en Secundaria. Una experiencia española. México, SEP/Cooperación española. Biblioteca para la Actualización del Maestro.

Consejo Mexicano de Investigación Educativa (comie) (2013) La investigación en México en el campo Educación y valores. 2002-2011. México, COMIE/ANUIES.

Conde, S. y Canedo, G. (coords.) (2004) El papel del directivo, del supervisor y del asesor Técnico pedagógico como promotores de ambientes escolares democráticos en la escuela primaria. Antología. México, IFE.

Cullen, C. (2004) Autonomía moral, participación democrática y cuidado del otro. Buenos Aires, Centro de Publicaciones Educativas y Material Didáctico.

Gobierno Federal (2012) Sistema Educativo de los Estados Unidos Mexicanos. Principales cifras. Ciclo escolar 2011-2012. México, SEP, 77-81.

Hersh, R.; Reimer, J. y Paolitto, D. (1988) El crecimiento moral de Piaget a Kohlberg. Madrid, Narcea. 
LATAPí, P. (2003) El debate sobre los valores en la escuela mexicana. México, FCE.

Magendzo, A. (2005) Curriculum. Educación para la democracia en la modernidad. Santa Fe de Bogotá, Instituto para el desarrollo de la democracia 'Luis Carlos Galán'.

Magendzo, A. (2008). Pensamiento e ideas-fuerza de la educación en derechos humanos en Iberoamérica. Chile, unESCO/OEI/SM.

Martínez, M. (2000). El contrato moral del profesorado. Condiciones para una nueva escuela. México, SEP/Desclée de Brouwer. Biblioteca para la Actualización del Maestro.

Rodríguez McKeon, L. (2009) La configuración de la formación ciudadana en la escuela. Análisis de un caso en educación secundaria. México, UPN.

SCHMelKes, S. (2004) La formación de valores en la educación básica. México, SEP.

Secretaría de Educación Pública (1993) Plan y Programas de estudio 1993. Secundaria. México, SEP.

Secretaría de Educación Pública (1999) Formación Cívica y Ética Secundaria. Programa de estudios comentado. México, SEP.

Secretaría de Educación Pública (2006a) Formación Cívica y Ética. Secundaria. Programas de estudio. México, SEP.

SeCRetaría de EduCACión Pública (2006b) Materiales interactivos para la asignatura Formación Cívica y Ética. Consultado el 15 de junio de 2014. http://telesecundaria.dgme.sep. gob.mx/ (NOTA: Debido al proceso de reforma curricular la página web se encuentra temporalmente inactiva).

Secretaría de Educación Pública (2010) La telesecundaria en México. Un breve recorrido histórico por sus datos y relatos. México, SEP.

Secretaría de Educación Pública (2011) Modelo educativo para el fortalecimiento de telesecundaria. México, SEP.

Secretaría de Educación Pública (2011) Formación Cívica y Ética. Secundaria. Programas de estudio. México, SEP.

Yurén Camarena, T. (2000) Formación y puesta a distancia. México, Paidós.

Yurén Camarena, T.; Navia, C. y Saenger, C. (coords.) (2005) Ethos y autoformación docente. Análisis de dispositivos para la formación de profesores. México, Pomares.

Yurén Camarena, T. y ARAújo, S. (2007) Calidoscopio: valores, ciudadanía y ethos como problemas educativos. México, Universidad Autónoma de Morelos. 